\title{
Using the small alignment index chaos indicator to characterize the vibrational dynamics of a molecular system: LiNC-LiCN
}

\author{
P. Benitez, ${ }^{1}$ J. C. Losada, ${ }^{1}$ R. M. Benito, ${ }^{1}$ and F. Borondo ${ }^{2,3}$ \\ ${ }^{1}$ Grupo de Sistemas Complejos and Departamento de Física y Mecánica, Escuela Técnica Superior de Ingenieros Agrónomos, \\ Universidad Politécnica de Madrid, E-28040 Madrid, Spain \\ ${ }^{2}$ Departamento de Química, Universidad Autónoma de Madrid, Cantoblanco, E-28049 Madrid, Spain \\ ${ }^{3}$ Instituto de Ciencias Matemáticas (ICMAT), Cantoblanco, E-28049 Madrid, Spain
}

(Received 30 April 2015; published 19 October 2015)

\begin{abstract}
A study of the dynamical characteristics of the phase space corresponding to the vibrations of the LiNC-LiCN molecule using an analysis based on the small alignment index (SALI) is presented. SALI is a good indicator of chaos that can easily determine whether a given trajectory is regular or chaotic regardless of the dimensionality of the system, and can also provide a wealth of dynamical information when conveniently implemented. In two-dimensional (2D) systems SALI maps are computed as 2D phase space representations, where the SALI asymptotic values are represented in color scale. We show here how these maps provide full information on the dynamical phase space structure of the LiNC-LiCN system, even quantifying numerically the volume of the different zones of chaos and regularity as a function of the molecule excitation energy.
\end{abstract}

DOI: 10.1103/PhysRevE.92.042918

PACS number(s): 05.45.Ac, 05.45.Pq, 95.10.Fh

\section{INTRODUCTION}

The vibrational dynamical processes that take place in a single isolated molecule, usually gas phase, are critical to determine its reactivity. Identifying the vibrational modes that are effectively coupled, typically by Fermi resonances [1] and how energy flows among them [2,3], is a central point in molecular dynamics [4].

For two-dimensional (2D) systems there are well established mathematical tools to study this underlying dynamics. Poincaré surfaces of section (PSOS) is probably the most straightforward and informative one, although there are other equally good possibilities, such as local Lyapunov exponents [5], fast Lyapunov indicator and variants [6,7], or the Kolmogorov-Sinai entropy [8,9], to obtain a picture of the associated dynamics. Another possibility, based on the idea of analyzing the separation of nearby orbits, is the calculation of small alignment index (SALI) [10-28], which relies on the behavior of the associated position vectors in the phase space, which can be easily calculated from the (usually numerical) integration of Hamilton equations. The value of the SALI decreases exponentially to zero with time when the trajectory is chaotic. The great advantage of SALI is that the associated computational burden is largely independent of the dimensionality of the system [29]. The results obtained with these methods can be rationalized using the celebrated theorems due to Kolmogorov, Arnold and Moser (KAM) [30] and to Poincaré and Birkhoff (PB) [31], describing respectively the tori destruction, and the fate of resonant tori due to perturbations in Hamiltonian systems. In a Hamiltonian system with $N$ degrees of freedom, the motion is integrable if there are $N$ independent constants of the motion. A transformation to action-angle variables clearly shows that in this case trajectories lie on the surfaces of invariant tori in phase space. These trajectories can be quasiperiodic if all frequencies are incommensurate, quasiperiodic on reduced dimensionality tori if some of them are in resonance, or one-dimensional periodic orbits (PO) if the resonance condition involves all frequencies. The KAM theorem establishes that, when such an integrable system is perturbed, some tori are destroyed but others persist (the so-called KAM tori). The fate of the destroyed ones is especially interesting in the case of resonant tori, since then an odd number of POs, half of them stable and the other half unstable, survive (PB theorem) forming a chain of islands structure surrounded by bands of stochasticity [32]. The nonresonant tori which are not "irrational enough" (in the sense of the KAM theorem) are also destroyed, turn into fractal objects called cantori [33,34].

The SALI indicator has been mainly used to distinguish between chaos and regular orbits in symplectic maps $[10,11,15,24]$, model Hamiltonians such as HénonHeiles [10-12], celestial mechanics problems [20,21], nonlinear lattices [15,16], condensate Bose-Einstein vortices [27], particle accelerators [13], galaxy models [19,22,23,25,26], and confined microplasma [28]. However this indicator has not yet been applied to the study of molecular vibrations. In this paper we consider the system LiNC-LiCN that has been an attractive subject for computational work, since it constitutes an example of a simple isomerization reaction. It has been extensively studied by our group [35-37], and considered for reactivity control in the picosecond range [38,39] and also in coherent chemistry with $\mathrm{THz}$ pulses [40]. From a mechanical point of view, it can be seen as a vibrational Hamiltonian system, consisting of a collection of highly anharmonic, coupled oscillators with a highly nonlinear behavior. Indeed, the intramolecular vibrational relaxation leading and/or competing with reactivity renders a quite rich dynamics. This is due to a number of reasons. The potential interactions can be described quite realistically, and contain a number of elements contributing to a complicated dynamics.

LiNC-LiCN presents two stable isomers at the linear configurations $\mathrm{Li}-\mathrm{N}-\mathrm{C}$ (the most stable one) and $\mathrm{Li}-\mathrm{C}-\mathrm{N}$ (local minimum), which are separated by a relatively modest energy barrier of only $3434.0 \mathrm{~cm}^{-1}$. To describe the vibrational dynamics of the system we use in this paper a 2D model, in which the $\mathrm{C}-\mathrm{N}$ bond is hold frozen at its equilibrium 
distance of $r_{e}=2.186$ a.u. This allows convenient monitoring of its dynamics by PSOS. Moreover, this simplification is well justified since the $\mathrm{C}-\mathrm{N}$ bond frequency is very high, and then the corresponding motion adiabatically separates from the rest of the molecular vibrations in the molecule [41].

The motion in the bending angle is very floppy, and thus the Li atom easily explores ample, yet anharmonic, regions of the potential energy surface, even rotating around the $\mathrm{C}-\mathrm{N}$ fragment (isomerization reaction $\mathrm{LiNC} \leftrightharpoons \mathrm{LiCN}$ ). For this reason, chaos sets in at low values of the excitation energy.

The final goal of this manuscript is to establish a general methodology to measure the fraction of chaos and the degree of chaoticity in molecular systems with mixed phase space structure as a function of the total energy of the system. To this end, we construct different maps based on the SALI indicator which are suitable for the study of dynamical Hamiltonian systems of any dimensionality. We show that the methods that are introduced have a superb performance, being not only able to distinguish between regular and chaotic motions, but giving also valuable information about the latter. For example, in our case we show that there exist two types of chaos, which originate from the different underlying structure of the phase space. With this methodology we have also accurately estimated the fraction of phase space filled by these two types of chaos.

The organization of the paper is as follows. In Sec. II A, we present the model used to describe the LiNC-LiCN molecule vibrational dynamics, giving the corresponding Hamiltonian in Jacobi or scattering coordinates, and the analytical expression for the potential energy surface. In Sec. II B, we briefly discuss the calculation of trajectories and Poincaré surfaces of sections in the LiNC-LiCN molecular system. In Sec. II C, we present the calculational procedure involved in the SALI analysis. In Sec. II D, we introduce different SALI based maps and a derived indicator that will be used in this work. The results obtained in our study, consisting of some maps with support based on phase space and colored with the corresponding values of the SALI, are presented and discussed in Sec. III. Finally, in Sec. IV we summarize the main conclusions derived from this work.

\section{SYSTEM AND CALCULATIONS}

\section{A. The LiNC-LiCN molecular system}

Using scattering or Jacobi coordinates $(R, \theta)$, where $R$ is the distance from the $\mathrm{Li}$ atom to the center of mass of the $\mathrm{C}-\mathrm{N}$ fragment, and $\theta$ is the angle between the $\mathrm{C}-\mathrm{N}$ and $R$ vectors, the $2 \mathrm{D}$ reduced classical vibrational (rotationless) Hamiltonian for the LiNC-LiCN molecule is given by

$$
H=\frac{P_{R}^{2}}{2 \mu_{1}}+\frac{1}{2}\left(\frac{1}{\mu_{1} R^{2}}+\frac{1}{\mu_{2} r_{e}^{2}}\right) P_{\theta}^{2}+V(R, \theta),
$$

where $\mu_{1}=m_{\mathrm{Li}}\left(m_{\mathrm{C}}+m_{\mathrm{N}}\right) /\left(m_{\mathrm{Li}}+m_{\mathrm{C}}+m_{\mathrm{N}}\right)$ is the $\mathrm{Li}-\mathrm{CN}$ reduced mass, and $\mu_{2}=m_{\mathrm{C}} m_{\mathrm{N}} /\left(m_{\mathrm{C}}+m_{\mathrm{N}}\right)$ is the $\mathrm{C}-\mathrm{N}$ reduced mass.

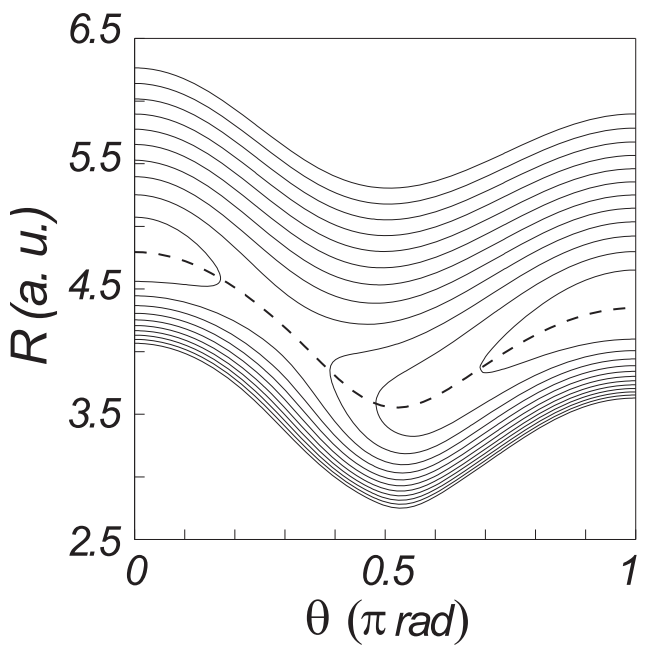

FIG. 1. Contours plot of the LiNC-LiCN potential energy surface, $V(R, \theta)$. The minimum energy path, $R_{e}(\theta)$, connecting the two stable isomers, $\operatorname{LiCN}(\theta=0)$ and $\operatorname{LiNC}(\theta=\pi \mathrm{rad})$, is shown as a dashed line.

In our model, the atomic interactions are described by means of a realistic potential energy surface, $V(R, \theta)$, which consists of an expansion in Legendre polynomials

$$
V(R, \theta)=\sum_{\lambda=1}^{9} a_{\lambda}(R) P_{\lambda}(\theta),
$$

where the coefficients, which have been fitted to $a b$ initio quantum mechanical calculations, have been taken from the literature [42]. This function has two wells at the linear configurations $\theta=0$ for the isomer $\mathrm{Li}-\mathrm{CN}$ and $\theta=\pi$ for Li-NC. It is presented in Fig. 1 in the form of a contour plot, along with the minimum energy path (MEP), $R_{e}(\theta)$, connecting the two wells.

\section{B. Classical trajectories and Poincaré surfaces of sections}

Classical trajectories for our system are calculated by numerical integration of the Hamilton equations of motion corresponding to Eq. (1). In this way we get a time series $\left(\theta, R, P_{\theta}, P_{R}\right)_{t}$ for each value of the initial conditions $\left(\theta, R, P_{\theta}, P_{R}\right)_{0}$ at a given vibrational energy, whose dynamics can be studied. The vibrational dynamics associated with the isomerization LiNC $\rightleftharpoons \mathrm{LiCN}$ process can be adequately followed by monitoring the motion along the angular $\theta$ coordinate [41]. For the sake of comparison in this work we will use, in addition to the SALI indicator, the more established procedure of PSOS specifically to select initial conditions in the phase space [35-37].

For each trajectory a PSOS is computed by taking the sectioning coordinate to lie along the minimum energy path connecting the two isomers [36], which is given by the analytical expression

$$
\begin{aligned}
R_{e}(\theta)= & 4.1159+0.25510 \cos \theta+0.49830 \cos 2 \theta \\
& +0.053427 \cos 3 \theta-0.068124 \cos 4 \theta \\
& +0.020578 \cos 5 \theta
\end{aligned}
$$


in atomic units. In order to make this PSOS an area preserving map, the following canonical transformation [36] is performed:

$$
\begin{gathered}
\rho=R-R_{e}(\theta), \quad \psi=\theta, \\
P_{\rho}=P_{R}, \quad P_{\psi}=P_{\theta}+\left(\frac{d R_{e}}{d \theta}\right)_{\theta=\psi} P_{R} .
\end{gathered}
$$

In this way the PSOS is defined as $\rho=0, P_{\rho}$ being obtained from the energy conservation condition given by Eq. (1) and taking the branch of the negative discriminant in the resultant quadratic equation.

This PSOS has the symmetry property

$$
\psi \rightarrow 2 \pi-\psi, \quad P_{\psi} \rightarrow-P_{\psi},
$$

so that only the interval $0 \leqslant \psi \leqslant \pi$ needs to be represented in the corresponding plots.

\section{SALI calculation}

Given a reference trajectory, the SALI index considers the behavior of the vectors associated with nearby trajectories, that can indeed be easily calculated from the results obtained from trajectory propagation. As indicated before, it can be used in any system to ascertain the dynamical characteristics of the trajectories, regardless of the involved number of dimensions [10-12,14,18,29]. The procedure is as follows. Let us consider $z \overrightarrow{(t)}=\left(q_{1}(t), \ldots, q_{N}(t), p_{1}(t), \ldots, p_{N}(t)\right)$ to be the reference trajectory in the phase space, and $\vec{z}_{1}(t), \vec{z}_{2}(t)$ two nearby trajectories (see Fig. 2). Then we define the corresponding deviation vectors $\vec{v}_{i}$ as

$$
\vec{v}_{i}(t)=\vec{z}(t)-\vec{z}_{i}(t), \quad i=1,2,
$$

with the associated unitary vectors

$$
\hat{v}_{i}(t)=\frac{\vec{v}_{i}(t)}{\left|\vec{v}_{i}(t)\right|}, \quad i=1,2 .
$$

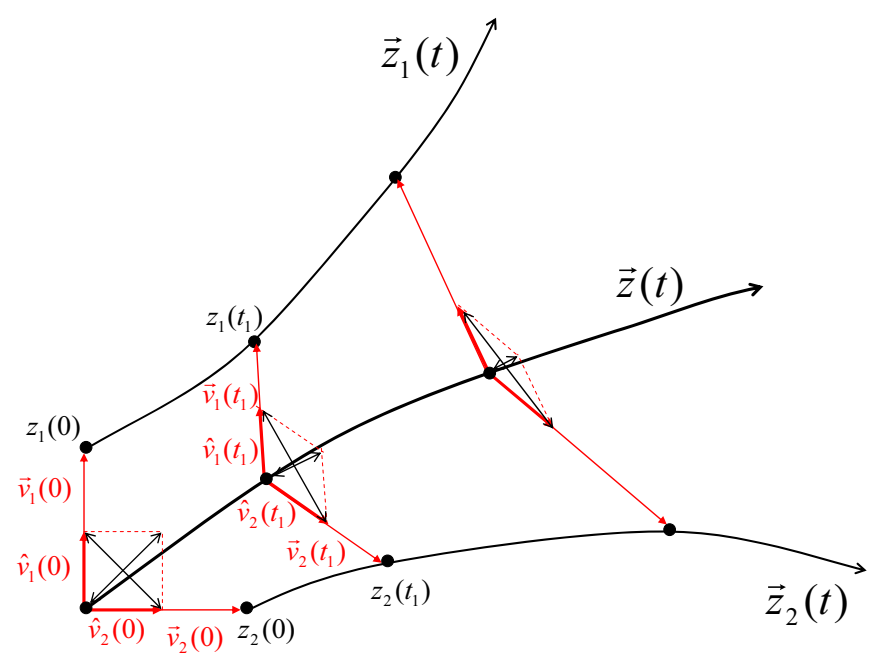

FIG. 2. (Color online) Graphical illustration of how the sum and difference of two vectors near a reference trajectory are calculated.
Trajectories $\vec{z}(t)$ and $\vec{z}_{i}(t), i=1,2$, derive from a hamiltonian and then

$$
\begin{gathered}
\frac{d \vec{z}(t)}{d t}=J \vec{\nabla} H(\vec{z}(t)), \\
\frac{d \vec{z}_{i}(t)}{d t}=J \vec{\nabla} H\left(\vec{z}_{i}(t)\right), \quad i=1,2,
\end{gathered}
$$

where the matrix $J$ is given by

$$
J=\left[\begin{array}{cc}
0_{N} & I_{N} \\
-I_{N} & 0_{N}
\end{array}\right]
$$

Combining Eq. (6) and Eq. (9), we have:

$$
\frac{d\left(\vec{z}(t)-\vec{v}_{i}(t)\right)}{d t}=J \vec{\nabla} H\left(\vec{z}(t)-\vec{v}_{i}(t)\right), \quad i=1,2 .
$$

Now taking Eq. (8) into account, we can derive [11]

$$
\frac{d \vec{v}_{i}(t)}{d t}=J \nabla^{2} H(\vec{z}(t)) \vec{v}_{i}(t)
$$

Then, by simultaneously solving Eqs. (8) and (12) for a given set of initials conditions $\left(z_{0}, v_{1,2}^{0}\right)$, both the reference trajectory and the associated values of the deviation vectors of the two nearby orbits are obtained. As can be seen in Fig. 2, the form of rhomboid formed by the two deviation unitary vectors changes with time, in such a way that when there is an exponential separation of the trajectories, i.e., chaos, the distance in one of the axes quickly goes to zero. The SALI indicator [10] is then defined as

$$
\operatorname{SALI}\left(z_{0}, t\right)=\min \left(\left|\hat{v}_{1}(t)+\hat{v}_{2}(t)\right|,\left|\hat{v}_{1}(t)-\hat{v}_{2}(t)\right|\right) .
$$

It has been shown $[10,11]$ that for a chaotic trajectory $\operatorname{SALI}\left(z_{0}, t\right) \sim e^{-\lambda t}, \lambda>0, \lambda=\lambda_{1}-\lambda_{2}$, where $\lambda_{1}, \lambda_{2}$ are the two primers' Lyapunov coefficients [11], while for a regular trajectory the value of this indicator oscillate around a constant value $\operatorname{SALI}\left(z_{0}, t\right) \sim O(1)$.

\section{SALI maps}

$\operatorname{SALI}\left(z_{0}, t\right)$ is an indicator able to discern between a regular and a chaotic trajectory by looking at its time evolution. In order to get a global visualization of the trajectories of the system we define a picture of the character map by assigning a value of the SALI indicator to each trajectory. To this purpose we choose the minimum value of $\operatorname{SALI}\left(z_{0}, t\right)$ in a given interval of time $\left(\mathrm{mSALI}_{T}\right)$. Then the map is defined by

$$
\begin{aligned}
\operatorname{mSALI}_{T}: \Omega & \rightarrow S, \\
\operatorname{mSALI}_{T}\left(z_{0}\right) & =\min \left\{\operatorname{SALI}\left(z_{0}, t\right)\right\}, \quad t \in(0, T], \\
S & =[0, \sqrt{2}],
\end{aligned}
$$

where $\Omega$ is the set of trajectories, $S$ is the closed interval $[0, \sqrt{2}]$ of valid values, and $T$ is the time interval considered. In order to be able to compare the results obtained from the SALI maps with those coming from (the more standard) PSOS, we obtain maps in which each point is colored by its $\mathrm{mSALI}_{T}$ value in a scale of colors.

We define then two kind of maps.

(1) First, the Poincare surface of section colored by SALI (SALI-PSOS map) results from marking all points of the 
classical PSOS generated from a given trajectory with the same color code corresponding to the $\mathrm{mSALI}_{T}$ defined above.

(2) Second, the SALI map (SM) results from coloring a grid of initial conditions (on the corresponding PSOS) according to the $\operatorname{mSALI}_{T}$ value for each trajectory defined by its initial condition value $z_{0}$.

Notice that these two possibilities are different, even if one chooses $z_{0}$ on the PSOS. Indeed, the first option contains more information, and it should be more useful when comparing with other chaos indicators, while the second one allows a straightforward generalization to systems with more than 2D.

\section{E. Numerical integration procedure}

Equations (8) and (12) have been solved by numerical integration. We have used a combination of a Runge-Kutta fourth-order method [43] for the first sixteen integration steps followed by a Gear method [44]. In the application presented in Sec. III the results shown have a $10^{-13}$ accuracy in the energy conservation. Some tests with another numerical integration methods have been carried out, finding similar results [45].

\section{RESULTS AND DISCUSSION}

In Fig. 3 we show together the standard PSOS and the SALI-PSOS maps for the 2D model of LiNC-LiCN described in Sec. II A for three representative values of the vibrational energy, namely $E=1510.5,2549.2$, and $3823.8 \mathrm{~cm}^{-1}$, for comparison. In both cases, the trajectories are started on a fine mesh of initial conditions on the PSOS and propagated for the same amount of time $\left(T=2.5 \times 10^{5}\right.$ a.u $)$.

In the first case, corresponding to the relatively low value of $E=1510.5 \mathrm{~cm}^{-1}$, we see in the PSOS that the dynamics are regular, taking place around the well corresponding to the most stable isomer, $\mathrm{Li}-\mathrm{NC}$. In the middle row, the results for a much larger value of the energy, $E=2549.2 \mathrm{~cm}^{-1}$, are shown. Here, motion in the less stable isomer, $\mathrm{Li}-\mathrm{CN}$, is classically accessible, and there are large regions of the phase space corresponding to the $\mathrm{Li}-\mathrm{NC}$ well, in which the dynamics are chaotic. Moreover, in the chaotic region one can see a noticeable accumulation of points next to the regular region in the $\mathrm{Li}-\mathrm{NC}$ well, which are clearly separated from the remaining, less populated, chaotic area. The separation between these two parts of the chaotic sea is a cantorus, whose origin and characteristics were thoroughly discussed in Ref. [46]. Finally, in the bottom panel, a larger energy of $3823.8 \mathrm{~cm}^{-1}$ is considered. Here, the dynamics become even more chaotic and isomerizing orbits connecting the two potential wells, in which the $\mathrm{Li}$ atom rotates around the $\mathrm{C}-\mathrm{N}$ fragment, do exist. The effect of the cantorus is still visible and a small island of stability born in the border of the inner region appears [46].

The corresponding results in the SALI-PSOS maps (right column) provide essentially the same information. Indeed, at the three energies considered, the lighter region (orange) $\left(\mathrm{mSALI}=10^{0}-10^{-4}\right)$ in both wells is predominant, indicating that the dynamics there are regular. (Areas in white indicate that no trajectories have been chosen inside them, similarly to what happens in the white regions in the PSOSs.) On the other hand, as increasing values of the energy are considered,
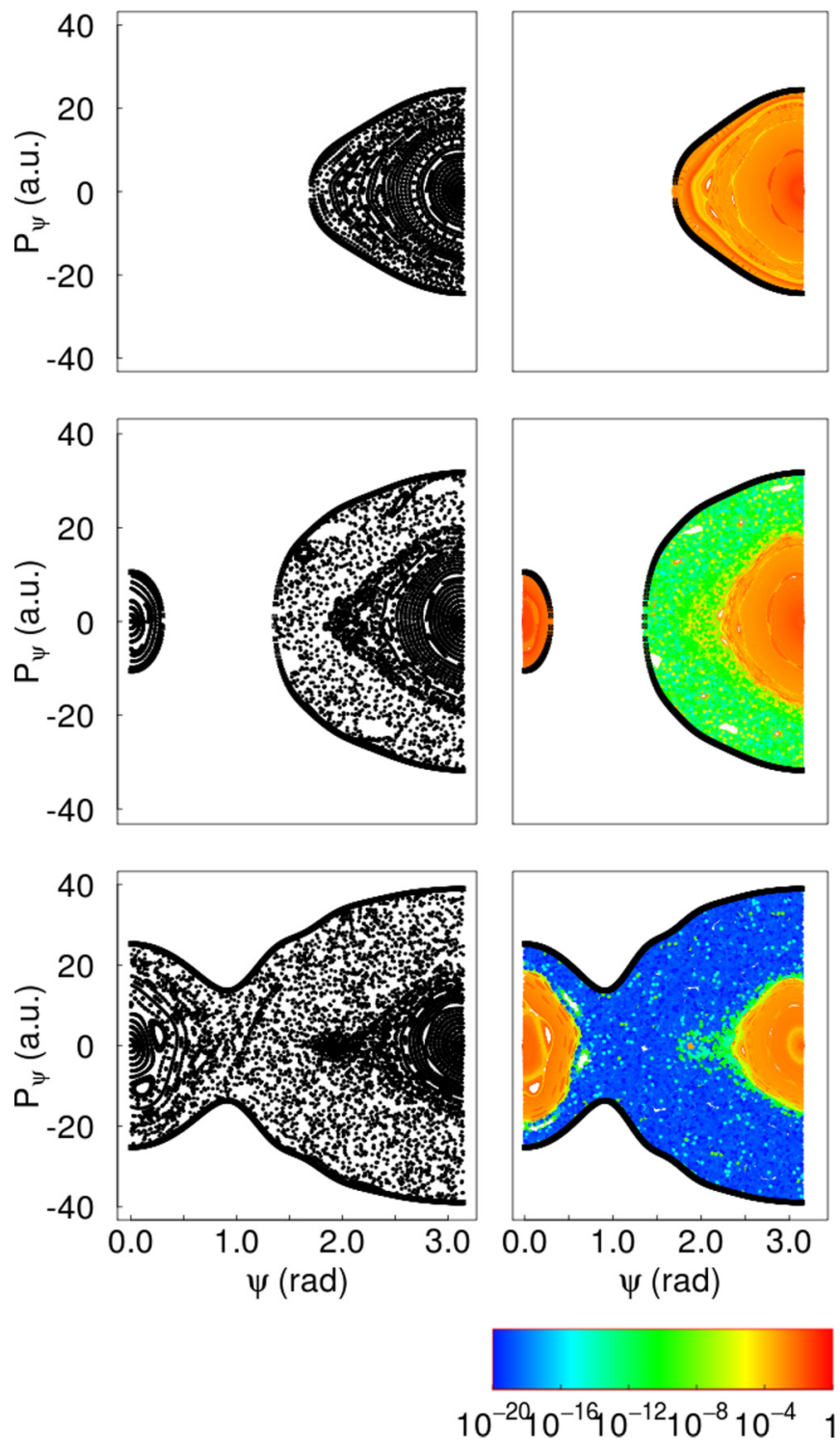

FIG. 3. (Color online) Composite Poincaré surfaces of sections (PSOSs) (left column) and the corresponding PSOSs colored by SALI (SALI-PSOSs) (right column) at different values of the vibrational energy: $1510.5^{-1}$ (top), 2549.2 $2^{-1}$ (middle), and 3823.8 $\mathrm{cm}^{-1}$ (bottom). The logarithmic scale of colors, corresponding to the $\mathrm{mSALI}$ values, is also shown in the bottom right corner of the figure.

more darker areas (in green and blue) are visible, due to the fast decrease in the mSALI ( $\sim 10^{-6}$ and $\sim 10^{-18}$, respectively) which is associated with chaotic motion. As can be seen, these colors appear respectively in the same places of phase space that KAM tori or dispersed points appear in the corresponding PSOS. However, the SALI maps shows here a richer spectrum of colors, that not only separates regular from chaotic orbits [18] but also allows the distinction among different degrees of chaoticity in the motion. Indeed, in the regular regions tori or chains of islands with their regions of stable and unstable motion appear in slightly different colors (see for example the upper right panel in Fig. 3), and in the chaotic areas the existence of cantori are easily spotted by an abrupt change in color [from orange, in the well region, to 

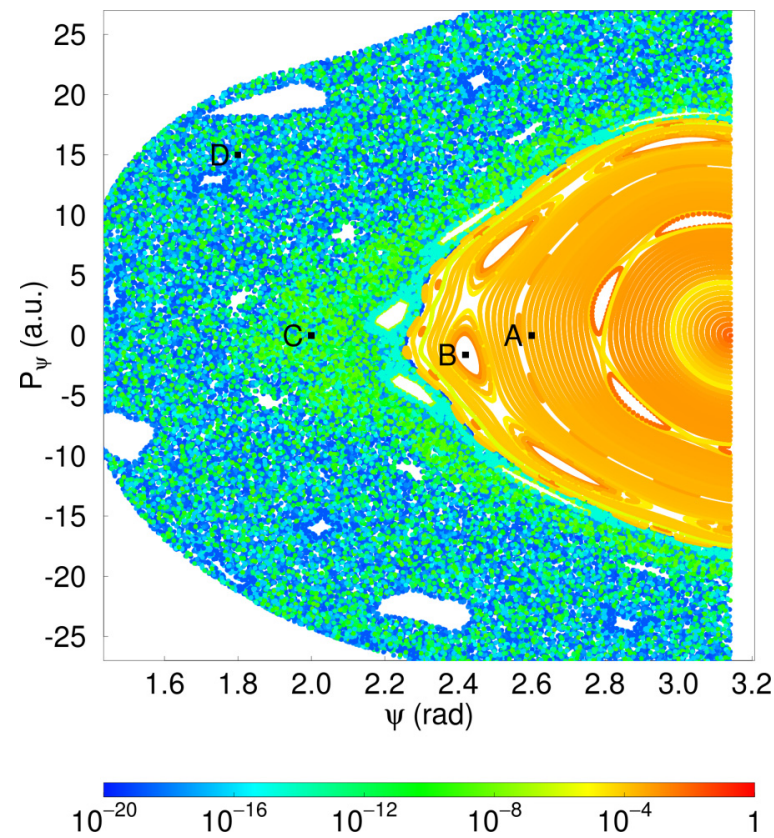

FIG. 4. (Color online) Blow-up of the SALI-PSOS map in the middle panel of Fig. 3. The initial positions (A-D) of the four orbits discussed in the text (see also Table I) are shown.

green in the outer part of the middle right panel of Fig. 3, and from green (light) to blue (dark) in the bottom right one]. A more detailed picture of this effect is shown in Fig. 4.

\section{A. Time evolution of SALI}

\section{Time evolution of SALI for trajectories with different dynamical characteristics}

To discuss in more detail the last point of the previous subsection, we select in the SALI-PSOS corresponding to $E=$ $2549.2 \mathrm{~cm}^{-1}$ four trajectories with very different dynamical characteristics. They are marked in Fig. 4 with letters A-D, and their initial conditions and characteristics are summarized in Table I. The corresponding orbits in configuration space, PSOSs, and time evolution of the SALIs are shown in the three top rows of Fig. 5. Since this last magnitude is strongly oscillating, we also present in the bottom row a time averaged version $(\overline{\mathrm{SALI}})$ over a time window $\tau$ given by

$$
\overline{\operatorname{SALI}}\left(z_{0}, t\right)=\int_{-\tau / 2}^{\tau / 2} \operatorname{SALI}\left(z_{0}, t+\eta\right) d \eta,
$$

which shows more clearly the overall tendency. In our calculations we use a window of width $\tau=3.5 \times 10^{3}$ a.u.

TABLE I. Initial conditions and dynamical characteristics for the four trajectories marked in Fig. 4 and shown Fig. 5.

\begin{tabular}{lcc}
\hline \hline Traj. & $z_{0}=\left(\psi_{0}, P_{\psi}^{0}\right)(\mathrm{rad}$, a.u. $)$ & Dynamical character \\
\hline $\mathrm{A}$ & $(2.60,0)$ & Quasiperiodic \\
$\mathrm{B}$ & $(2.4232351,-1.6184869)$ & Stable periodic \\
$\mathrm{C}$ & $(2.0,0)$ & Chaotic inside cantorus \\
$\mathrm{D}$ & $(1.80,15.0)$ & Chaotic \\
\hline \hline
\end{tabular}

An examination of the results in Fig. 5 indicates that trajectories A and B correspond to regular orbits. As can be seen in the plots in the first two rows, the first one (A) is quasiperiodic, leaving in the PSOS a closed curve resulting from the intersection of the associated invariant torus with $\rho=0$. The second one (B) on the other hand is a periodic orbit that leaves four fixed points [eight if we do not take into account the symmetry in Eq. (5)] which are visited periodically by the orbit.

The corresponding values of SALI and $\overline{\text { SALI }}$ (third and fourth rows in the figures respectively) are high at all times explored and approximately constant.

On the other hand $\mathrm{C}$ and $\mathrm{D}$ correspond to irregular looking orbits, which both give rise to a cloud of points in the PSOS filling the available chaotic phase space. The corresponding values of SALI and $\overline{\text { SALI }}$ decrease quickly to zero, in agreement with the chaotic character of the trajectories. However, when closely examined one sees that these two trajectories behave rather differently. While in case $\mathrm{C}$ the final value of SALI is $\sim 10^{-4}$, in case $\mathrm{D}$ this value decreases to $\sim 10^{-10}$ in the same time.

More specifically, for trajectory $\mathrm{C}$ the value of SALI initially does not change significantly during a certain interval of time (of the order of $O=10^{5}$ a.u.), giving rise to some sort of plateau where its value remains approximately stationary. This behavior is the equivalent to the accumulation of points observed in the PSOS, and it is due to the fact that the initial condition chosen in this case (see Table I) is very close to the LiNC-LiCN regular region. This type of regions are known [34] to be dynamically "sticky," temporarily trapping neighbor orbits. Moreover, this effect is reinforced in this case by the bottleneck effect [33] induced by the presence of a cantorus. During this period of time, the trajectory behaves as if regular, and that is why its SALI practically does not decrease. There are also other less prominent plateaus both in the time evolution of the SALI for orbits C and D. They are due to the periods of time in which the trajectories get close to the almost regular structures near the cantorus, thus being affected by its dynamical influence.

\section{Local behavior of a global ergodic trajectory influenced by a cantorus}

Let us consider next in more detail the information provided by SALI regarding trajectory $\mathrm{C}$, which is chaotic, but starts inside the cantorus and is close to the LiNC-LiCN regular region. For this purpose we have propagated the orbit for a much longer time, actually $1.3 \times 10^{6}$ a.u. The results are shown in Fig. 6, where it can be seen that, after the initial plateau shown in red, the SALI decreases very quickly, as it should for a chaotic trajectory, until $t \sim 5 \times 10^{5}$ a.u. is reached, where it stabilizes, nevertheless presenting some obvious oscillations. To further investigate the reason for the different behaviors in this curve we have selected different portions in it, one at the very beginning where the SALI increases and stabilizes, a second one in which it decreases quickly, and a third one on the final stabilization plateau. They have been marked by (a) red, (b) blue, and (c) green, respectively. The corresponding portions of the whole orbit in configuration space, in phase space PSOS, and the time 
A
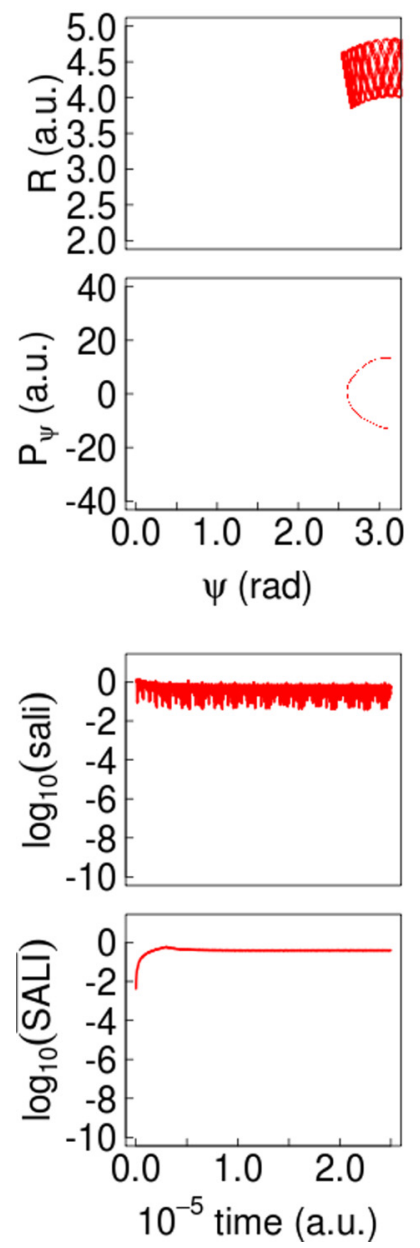

B
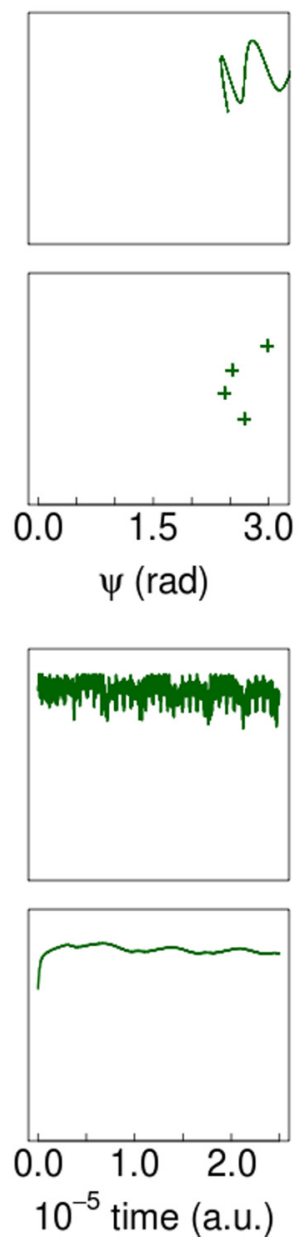

C
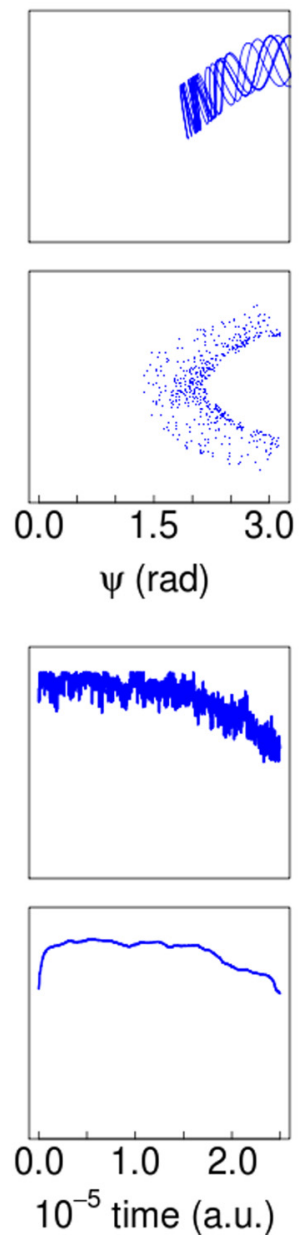

D
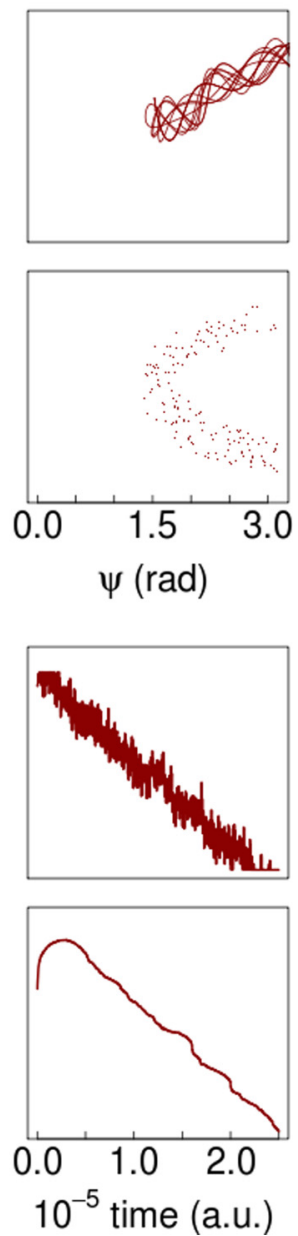

FIG. 5. (Color online) Orbit in configuration space (top row), Poincaré surface of section (second row), and time evolution of the actual SALI (third row) and its averaged version, $\overline{\mathrm{SALI}}$, for the four trajectories A-D marked in Fig. 4 (see also Table I and text for details).

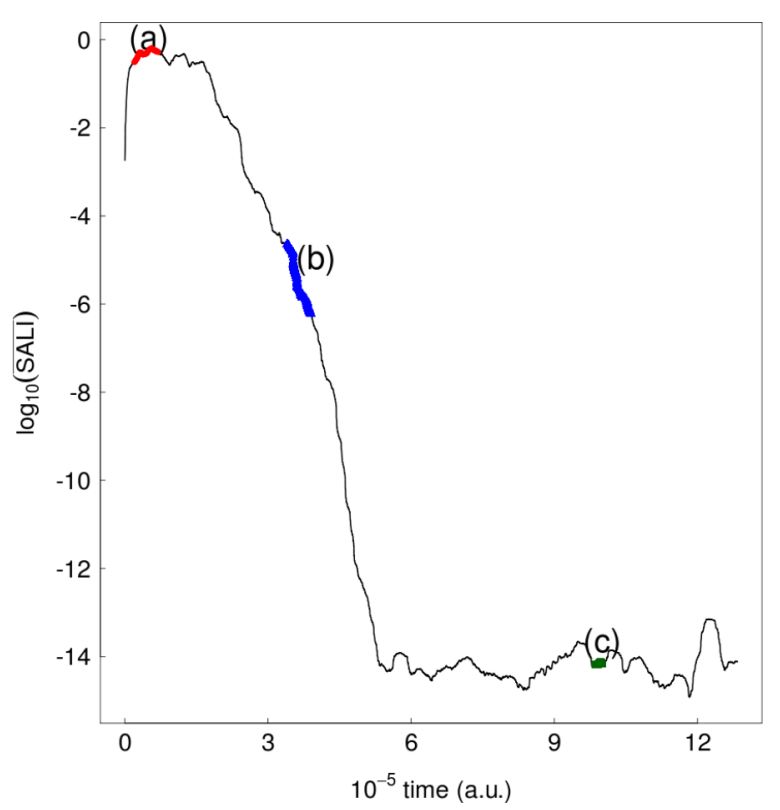

FIG. 6. (Color online) Time evolution of SALI for the chaotic trajectory $\mathrm{C}$ of Fig. 4 and described in Table I for $T=1.3 \times 10^{6}$ a.u. evolution of $\overline{\mathrm{SALI}}$ are shown in the different rows of Fig. 7. As can be seen, in the (a) and (c) intervals where the trajectory is close to the regular LiNC-LiCN region the value of SALI is (approximately) stationary, while in the (b) one, corresponding to the chaotic region, it decreases quickly. That is, the time evolution of the SALI gives a clear indication of those periods of time in which a (globally) ergodic trajectory is (locally) influenced by the regular or almost regular structures that exists in the mixed phase space of this molecular system $[47,48]$.

\section{SALI and the neighborhood of periodic orbits}

To further investigate the performance of SALI to discern among trajectories with different dynamical characteristics, we consider next a pair of stable $(S)$ and unstable $(U)$ periodic orbits, linked in the sense that they originate, due to the fate dictated by the PB theorem, from the same original resonant torus and its neighborhood. Here, we will see that the SALI can also be viewed more as an indicator of orbit stability than as an indicator of chaos.

For this purpose we have chosen the orbits marked in red $(S$ and $U)$ in Fig. 8. This figure is a blow-up of the composite PSOS for LiNC-LiCN at $E=2549.2 \mathrm{~cm}^{-1}$ in Fig. 4 corresponding to the region near trajectory $\mathrm{B}$. We have 

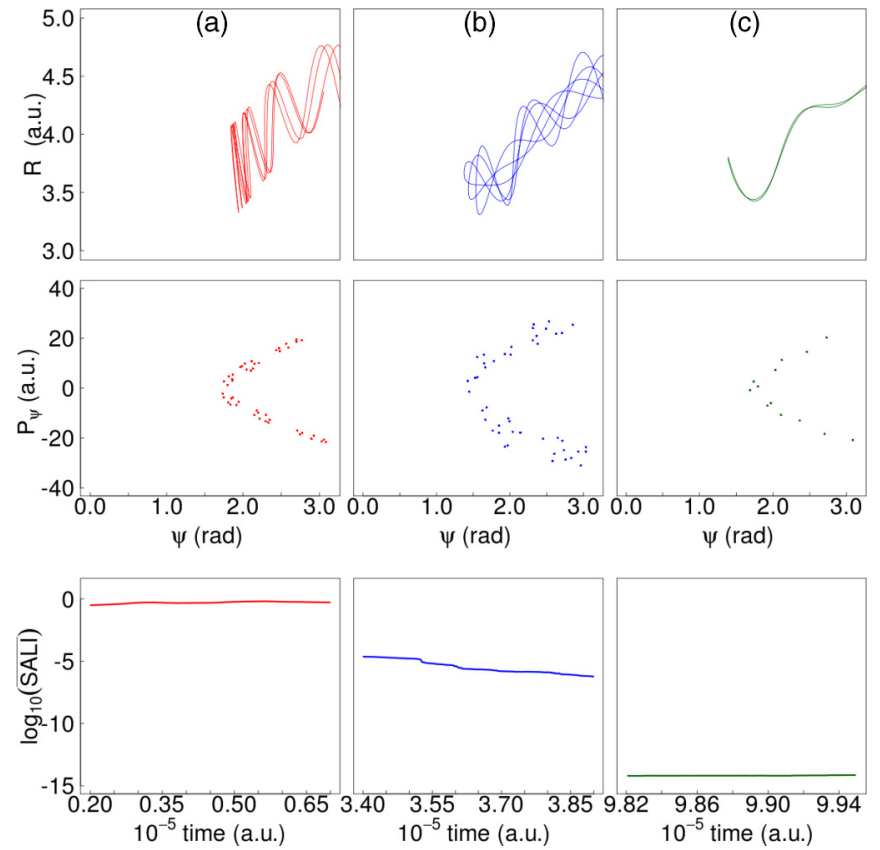

FIG. 7. (Color online) Orbit in configuration space (top), PSOS (middle), and time evolution of $\overline{\mathrm{SALI}}_{T}$ (bottom) for the different portions of trajectory $\mathrm{C}$ shown in Fig. 6.

also marked in green and blue, respectively, two trajectories, $U^{\prime}$ the $U^{\prime \prime}$, close to the unstable orbit and two more trajectories, $S^{\prime}$ and $S^{\prime \prime}$, marked in green and blue close to the stable orbit, making a total of six orbits.

The results corresponding to the stable choice are shown in Fig. 9, where we show together the time evolution of the $\overline{\text { SALI }}$ and the configuration space representation of the three orbits. Recall that these orbits are part of a chain of islands structure derived from the $\mathrm{PB}$ theorem.

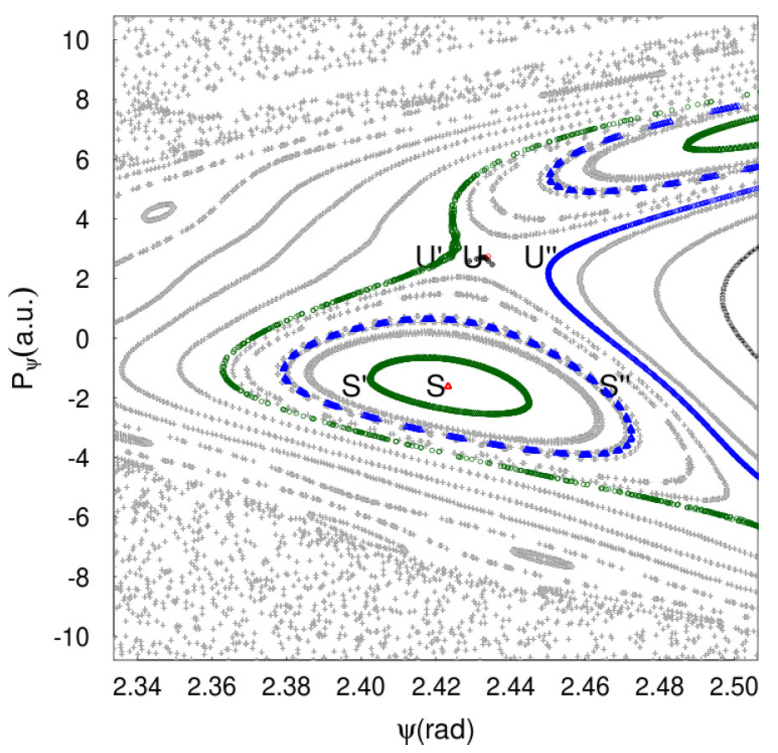

FIG. 8. (Color online) Blow-up of the composite Poincaré surface of section for LiNC-LiCN at $E=2549.2 \mathrm{~cm}^{-1}$ presented in Fig. 4 near trajectory B.
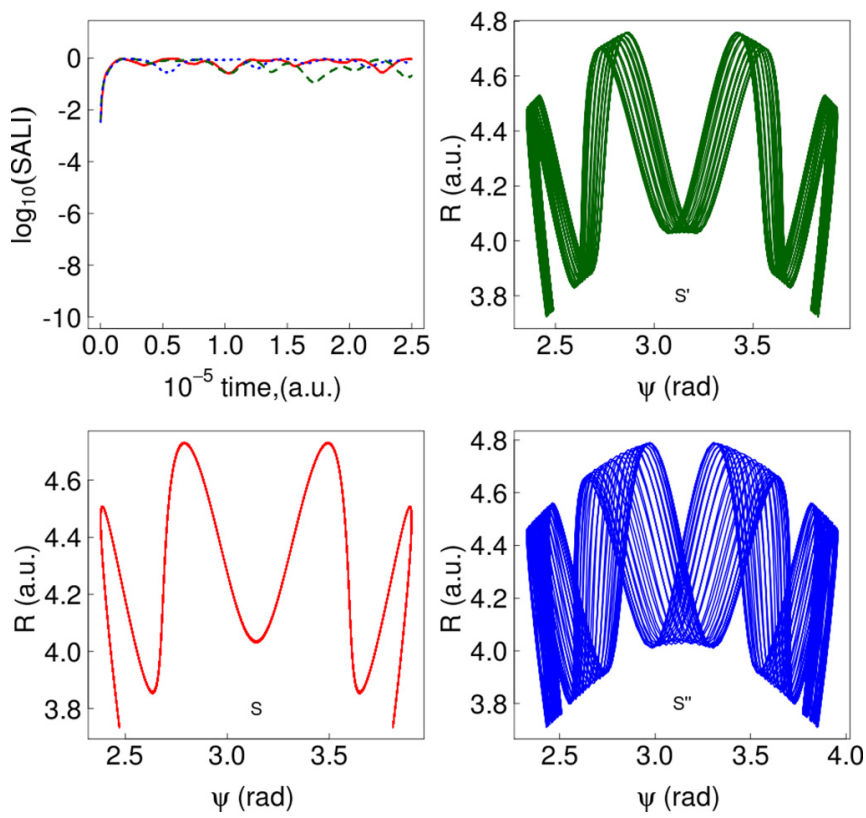

FIG. 9. (Color online) Time evolution of $\overline{\text { SALI }}$ (top left) for the orbits: stable $S$ (full red line) and its neighbors $S^{\prime}$ (dashed green line) and $S^{\prime \prime}$ (dotted blue line) marked in Fig. 8. See text for details. Orbits $S, S^{\prime}$, and $S^{\prime \prime}$ are plotted in configuration space.

Since all the orbits are in a regular region of phase space, the value of SALI oscillates around a practically constant high value (of the order of $\sim 10^{-0.25}=0.56$ ).

The case of the region around the unstable PO is in principle a bit more complicated. The phase space immediate to the orbit is chaotic, and then SALI is expected to decrease quickly. On the other hand, chaos is here restricted to a narrow band of stochasticity structured through the homoclinic oscillations of the central orbit, outside of which the dynamics are regular. The final outcome of these two competing effects cannot be predicted a priori and the answer has to be obtained from numerical calculations. Such results are shown in Fig. 10. Notice that in this case the green dashed orbit $U^{\prime}$ is closer to the PO, and then it follows more or less the separatrix in this PB structure, while the blue dotted one $U^{\prime \prime}$ is further away, and then closer to the regular region of KAM tori surrounding this band of stochasticity. As a result, the behavior of SALI is different for these orbits. While for the unstable PO the behavior is a clear exponential decay, for the two neighboring trajectories SALI shows a much smoother variation, presenting small oscillations around the mean value, which is analogous at those found for the quasiperiodic orbit A in Fig. 5.

\section{B. Regularity and chaos as a function of the energy}

Since we have analyzed in the previous section the performance of SALI to discern among trajectories with different dynamical characteristics, we will now use SALI to obtain a good characterization of phase space. This investigation has been carried out in two ways at a global level as a function of the vibrational energy. 

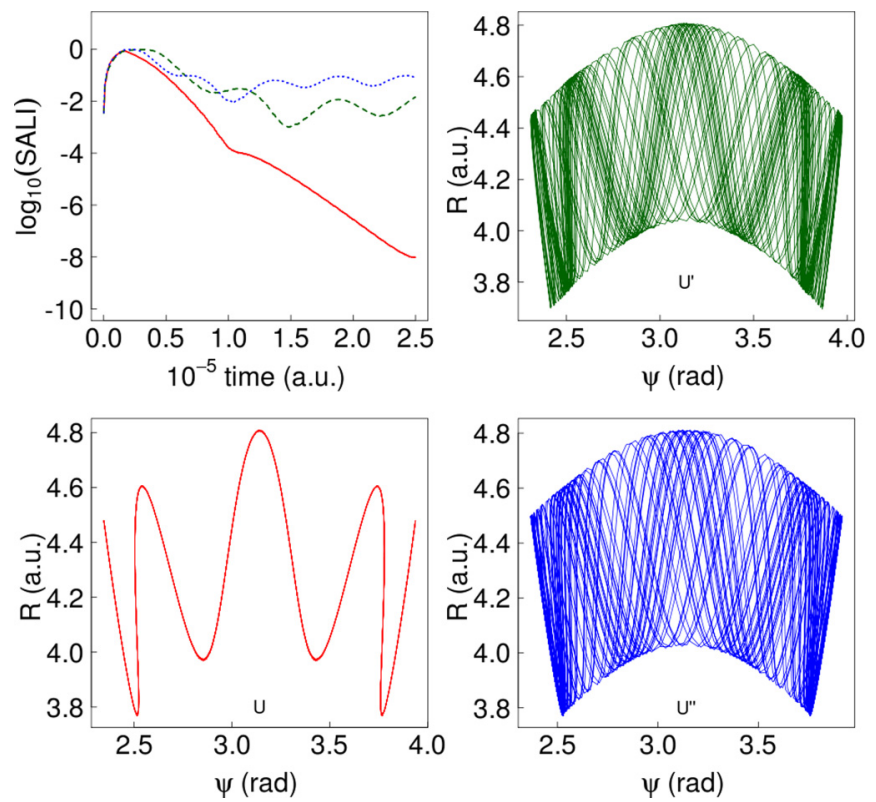

FIG. 10. (Color online) Time evolution of $\overline{\text { SALI }}$ for the orbits: unstable $U$ (full red line) and their neighbors $U^{\prime}$ (dashed green line) and $U^{\prime \prime}$ (dotted blue line) marked in Fig. 8. See text for details. Orbits $U, U^{\prime}$, and $U^{\prime \prime}$ are plotted in configuration space.

\section{Bifurcation diagram colored by SALI}

First, we use the SALI maps defined in Sec. II D with the selection of initial conditions in the well corresponding to the most stable isomer LiNC, defined as

$$
\Omega_{1}(R)=\left\{\begin{array}{l}
\psi=\pi, \\
P_{R}=0, \\
P_{\psi}=f_{1}\left(\psi, P_{R} ; E\right) .
\end{array}\right.
$$

We plot the $R$ coordinate vs energy, obtaining in this way a diagram [49] of orbits colored by $\mathrm{mSALI}_{T}$.

We consider the range of initial conditions in Eq. (16) plotting in each of these points the color corresponding to the orbit (not necessarily periodic) propagated from that point (BDS-SALI map).

The corresponding results for $\mathrm{LiNC}-\mathrm{LiCN}$ are shown in Fig. 11 superimposed to the usual bifurcation-continuation diagram [50,51] presented in black dots. As can be seen, the values of $\mathrm{mSALI}_{T}$ are high (red-orange) in the regions where the dynamics are regular (low values of energy and extreme values of $R$ ). As the excitation energy increases (2000-3200 $\mathrm{cm}^{-1}$ ) there appear regions with lower values of mSALI $_{T}$ (green or light), which nevertheless have embedded convoluted regions of regularity (red, $\operatorname{mSALI}_{T} \simeq 1$ ) as new POs are born in bifurcations.

Moreover, for $E \gtrsim 3200 \mathrm{~cm}^{-1}$ a sudden transition to chaos is observed, with a preponderance of darker regions (blue), except at the highest and lowest values of $R$, where the motion continues being essentially regular (even at very high values of $E$ ). Notice that for these extreme values of $R$ the other coordinate $\psi$ will be close to the equilibrium value around the well $(\psi \sim \pi)$ due to energy conservation.
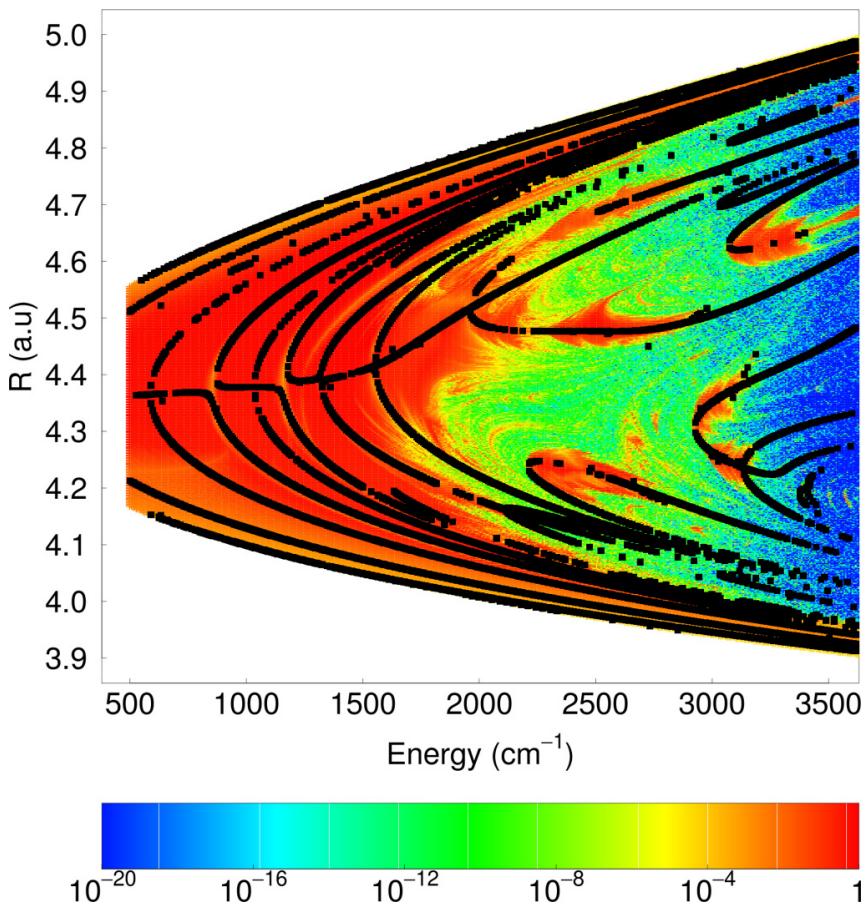

FIG. 11. (Color online) Bifurcation-continuation diagram colored by SALI for LiNC (BDS-SALI) as defined in Sec. III B 1. The grid of initial conditions $\Omega_{1}$ given in Eq. (16) is $\Delta R=2.2 \times 10^{-3}$ a.u. and $\Delta E=7.0 \mathrm{~cm}^{-1}$. The corresponding trajectories have been propagated up to $T=2.5 \times 10^{5}$ a.u. to obtain the values of $\mathrm{mSALI}_{T}$ in the plot using the color scale which is included in the figure. The usual bifurcation-continuation diagram of periodic orbits is also shown superimposed in black.

\section{SALI maps}

Next we consider the SM defined in Sec. II D but this time plotting the SALI values on a grid over the composite PSOS.

The corresponding choice of initial conditions is now

$$
\Omega_{2}\left(\psi, P_{\psi}\right)=\left\{\begin{array}{l}
\rho=0, \\
P_{\rho}=f_{2}(\psi, \rho ; E) .
\end{array}\right.
$$

The results for different values of the energy are shown in the left column of Fig. 12. To make this figure more quantitative, we have also plotted in the right column a histogram with the distribution of the different values of $\operatorname{mSALI}_{T}$ (in logarithmic scale), or equivalently their colors. As can be seen, for the lowest value of the energy considered, $E=1000 \mathrm{~cm}^{-1}$, all the points are in the yellow and orange range, with $\mathrm{mSALI}_{T}>10^{-6}$ and centered at $10^{-3}$ with more than $50 \%$ of the trajectories located in the corresponding bin. This is a clear indication that the dynamics are almost exclusively regular in this case.

As energy increases to $E=2000 \mathrm{~cm}^{-1}$ (second row), the available phase space grows and the new trajectories that appear in this region present smaller values of $\mathrm{mSALI}_{T}<$ $10^{-15}$, associated with colors in the green and blue range, which correspond to chaotic trajectories. Actually, we see the same distribution as before for $\operatorname{mSALI}_{T}>10^{-6}$ which correspond to the regular region, while new trajectories appear more or less evenly distributed in the rest of the values of $\operatorname{mSALI}_{T}$. 

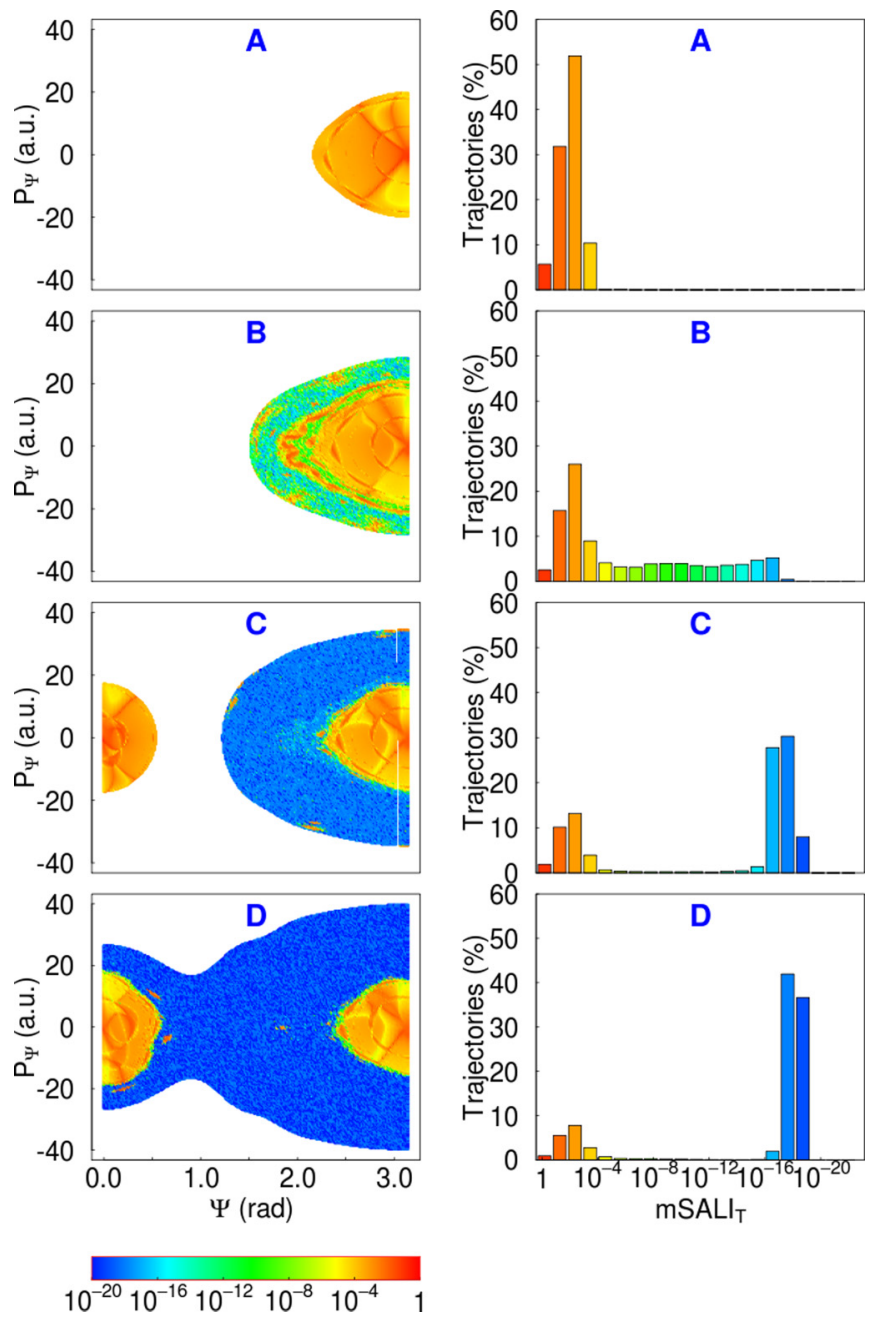

FIG. 12. (Color online) SALI Maps (left column) as defined in Sec. III B 1 for LiNC-LiCN at $E=1000 \mathrm{~cm}^{-1}, 2000 \mathrm{~cm}^{-1}, 3000$ $\mathrm{cm}^{-1}$, and $4000 \mathrm{~cm}^{-1}$ from A to D respectively. The grid of initial conditions $\Omega_{2}$ in Eq. (17) is $\Delta \psi_{0}=1.570 \times 10^{-2} \mathrm{rad}$ and $\Delta P_{\psi}^{0}=$ 0.4 a.u. The corresponding trajectories have been propagated up to $T=2.5 \times 10^{5}$ a.u. to obtain the values of $\mathrm{mSALI}_{T}$ in the plot using the color scale which is included in the figure. The corresponding histograms of $\mathrm{mSALI}_{T}$ are plotted in the right column. Notice that the last peak in panel D corresponds to SALI values lower than $10^{-19}$, and not only to the interval $\left(10^{-19}-10^{-20}\right)$ due to the accuracy limit of the computation.

Furthermore, when the energy increases to $E=3000 \mathrm{~cm}^{-1}$ (third row) the previous irregular trajectories have a larger phase space volume to roam, then become more chaotic. As a result the green colors disappear from the histogram moving towards the blue regions $\operatorname{mSALI}_{T}<10^{-16}$. Notice that the distribution of orange colors (regular motion) remains largely unchanged and still centered at $\mathrm{mSALI}_{T} \simeq 10^{-3}$, which is remarkable, especially if we take into account that a new region of stability around the $\mathrm{LiCN}$ isomer well opens up at this energy. This effect gets even more clear for the highest value of the energy considered, $E=4000 \mathrm{~cm}^{-1}$ (bottom row in the figure), where we see the increase of the blue peaks in the group of the chaotic trajectories, at the same time that the amount

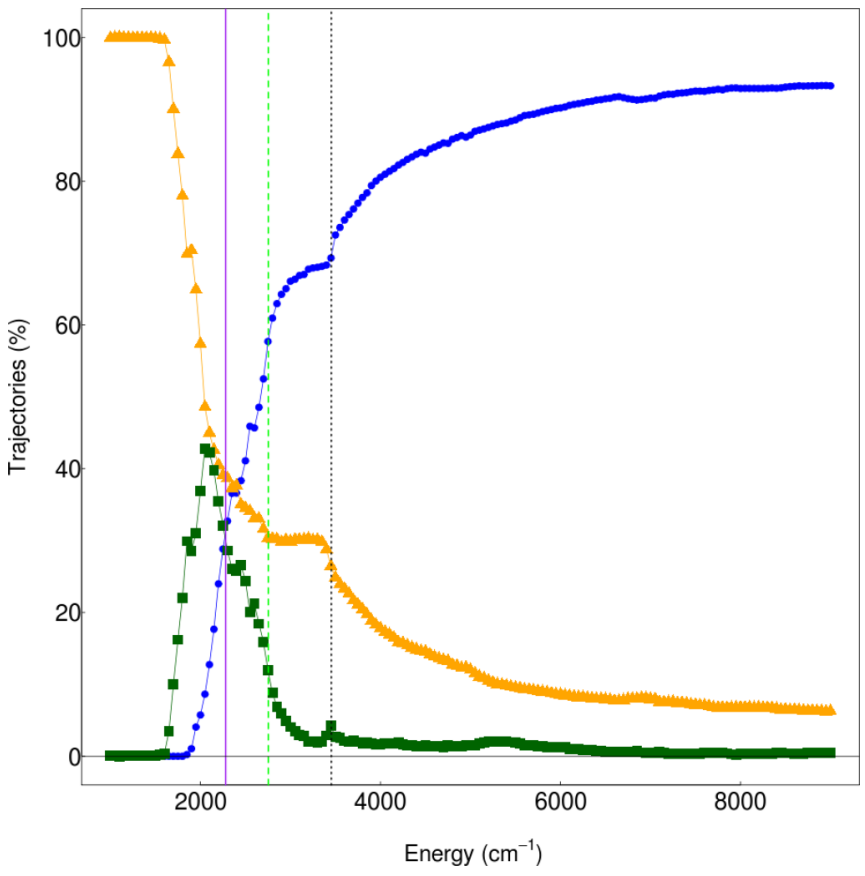

FIG. 13. (Color online) Fraction of the three different regions of the LiNC-LiCN mixed phase space corresponding to (orange triangles) regular motion $\left(\mathrm{mSALI}_{T}>10^{-5}\right)$, (green squares) mild chaos $\left(10^{-6}>\mathrm{mSALI}_{T}>10^{-16}\right)$, and (blue circles) strong chaos $\left(\mathrm{mSALI}_{T}<10^{-16}\right)$. The (purple) vertical line at $2281 \mathrm{~cm}^{-1}$ indicates the energy at which the region of the Li-CN isomer well begins to be accessible and motion in it starts. The (green) vertical line at $2754 \mathrm{~cm}^{-1}$ corresponds to the initial energy at which there is a compensation between the destruction of regular tori and the appearance of new regions of regularity. The (black) vertical line at $3450 \mathrm{~cm}^{-1}$ corresponds to the energy barrier for the isomerization reaction.

of regular trajectories have decreased (progressively from the smallest energies) here to a mere $10 \%$.

Summarizing, we conclude from our calculations that the mixed phase space of LiNC-LiCN consists of three different regions. One corresponds to the regular motions which are organized in KAM tori around the two existing isomer wells. A second one, of mild chaos, corresponds to irregular trajectories which are, however, influenced by the border of the above mentioned regular regions and other quasi-regular structures, such as cantori. A third region has strong chaos in which the trajectories move more freely, roaming in the ergodic part of phase space. Moreover, the proportion of these three regions changes with energy, going globally from more regular to more chaotic, as this perturbational parameter increases.

The effect is clearly illustrated in Fig. 13, where the percentage of these regions is represented as a function of the excitation energy. These percentages have been obtained by partitioning the trajectories in the SM histograms of Fig. 12 in the following three groups:

Regular motion: $\mathrm{mSALI}_{T}>10^{-6}$ (orange triangles)

Mild chaos: $10^{-6}>$ mSALI $_{T}>10^{-16}$ (green squares)

Strong chaos: $\mathrm{mSALI}_{T}<10^{-16}$ (blue circles)

As can be seen, for very low energies all the motion is regular and the fraction of regular dynamics (orange triangles) 
first remains almost constant up to $E=1550 \mathrm{~cm}^{-1}$, only showing a very small decrease in favor of the region of mild chaos. For $E>1550 \mathrm{~cm}^{-1}$ the fraction of regular dynamics suddenly drops linearly, due to an important destruction of tori (KAM theorem) giving rise to widespread chaos, until $E=2281 \mathrm{~cm}^{-1}$ (marked with vertical purple line in the figure) where the slope decreases due to the fact that at this value of the energy the motion on the region of the second isomer well, corresponding to regular motion, is classically accessible. Afterwards, the fraction of regular dynamics get constant at a value of $30 \%$ in the interval $2754-3450 \mathrm{~cm}^{-1}$ (between the green and black vertical lines), due to an apparent compensation between the destruction of regular tori in the LiNC and the appearance of regular motion in the $\mathrm{LiCN}$. Finally, the fraction of regular motion continues decreasing, this time in an exponential fashion, getting to an asymptotic value of $6 \%$. Notice that this value is nonzero, meaning that there is a resilient area of nondestructed regular motion even at very high values of the energy. The behavior of the fraction of strong chaos (blue circles) follows a similar pattern as before, but obviously presenting opposite tendencies. That is, up to $E=1550 \mathrm{~cm}^{-1}$ it is null, then it suddenly grows, in a linear fashion, up to $E=2281 \mathrm{~cm}^{-1}$ value of the energy where the slope decreases a bit, then the fraction of strong chaos becomes constant in the interval $E=2450-3450 \mathrm{~cm}^{-1}$, and finally this magnitude grows up to an asymptotic value of $93 \%$.

On the other hand, the fraction of mild chaos (green squares) behaves in a very different way to that of the region of strong chaos, presenting rather interesting characteristics. First, mild chaos only exists significantly in the interval $E=$ $1600-2900 \mathrm{~cm}^{-1}$. Second, mild chaos starts to grow significantly at the slightly lower value of energy as the strong chaos, then reaches its maximum of $21.08 \%$ at $E=1850 \mathrm{~cm}^{-1}$, going then rather quickly to zero after maintaining at a residual value of $\sim 0.6 \%$ between $E=2900$ and $9000 \mathrm{~cm}^{-1}$. This is the region of influence of the cantorus discussed in Sec. III, which temporarily traps trajectories inside it, which are then kept close to the border of regularity around the LiNC isomer, resulting in lower values of $\mathrm{mSALI}_{T}$, and then in mild chaos. As energy increases, the cantorus is progressively destroyed, making its role as a partial barrier for the flux of trajectories across weaker. Accordingly, the effect discussed above gets progressively less important, with the result that the fraction of mild chaos tends to be negligible, until only strong chaos is predominant in the ergodic region of phase space. This is shown by the two large peaks at the right side of panel D in Fig. 12.

One final comment is worth making here. Arriving at the interesting conclusions about the LiNC-LiCN mixed phase space structure discussed above has been possible due to the fact that an indicator so informative as the SALI has been used in our analysis.

\section{SUMMARY}

Summarizing, in this work we have presented a study on the application and performance of the small alignment index (SALI) to characterize the dynamics of molecular systems. This index is based on monitoring the separation of nearby trajectories, something which is done in a very efficient way, such that the computational burden involved is largely independent of the system dimensionality. Using the asymptotical numerical values of the SALI we have defined different illustrative maps, which allows an efficient and deep characterization of the dynamical characteristics of the system.

In particular, we have shown how SALI can not only discern between regular and chaotic trajectories, but can also provide extra information on the characteristics of the latter. For example, SALI can easily find the different behavior of trajectories in the vicinity of POs and cantori. This makes SALI a powerful tool in nonlinear dynamics, that goes beyond other more standard methods, such as PSOS, Lyapunov exponents, etc. Moreover, we have also shown how the SALI numerical values can be used in different diagram and maps, such as bifurcation diagrams colored by SALI or PSOS-SALI maps to get a global understanding of the phase space structure of a dynamical system.

As an example, we have applied the method to a realistic two-dimensional model for the vibrations of the LiNC-LiCN isomerizing system, that has been extensively studied in the past.

\section{ACKNOWLEDGMENTS}

This research was supported by the Ministerio de Economía y Competitividad (Spain) under Grants No. MTM2012-39101, and ICMAT Severo Ochoa No. SEV-2011-0087. We thank Prof. A. Jorba for his help and useful discussion concerning the integration methods.
[1] E. L. Sibert, W. P. Reinhardt, and J. T. Hynes, Classical dynamics of energy transfer between bonds in A B A triatomics, J. Chem. Phys. 77, 3583 (1982).

[2] S. Keshavamurthy, in Advances in Chemical Physics, Vol. 153, edited by S. A. Rice and A. R. Dinner (John Wiley \& Sons, Hoboken, 2013).

[3] M. Gruebele and P. G. Wolynes, Vibrational energy flow and chemical reactions, Acc. Chem. Res. 37, 261 (2004).

[4] The Chemical Bond: Structure and Dynamics, edited by A. Zewail (Academic Press, San Diego, 1992).

[5] Hamiltonian Systems with Three or More Degrees of Freedom, NATO ASI Series C, edited by C. Simó (Kluwer, Dordrecht, 1999).
[6] C. Froeschle and E. Lega, On the structure of symplectic mappings. The fast Lyapunov indicator: A very sensitive tool, Celestial Mech. Dyn. Astron. 78, 167 (2000).

[7] R. Barrio, Sensitivity tools vs. Poincaré sections, Chaos Solitons Fractals 25, 711 (2005).

[8] L. E. Reichl, The Transition to Chaos in Conservative Classical Systems: Quantum Manifestations (Springer, New York, 1992).

[9] Ya. G. Sinai, Introduction to Ergodic Theory (Princeton University Press, Princeton, 1977).

[10] Ch. Skokos, Alignment indices: A new, simple method for determining the ordered or chaotic nature of orbits, J. Phy. A: Math. Gen. 34, 10029 (2001). 
[11] Ch. Skokos, Ch. Antonopoulos, T. C. Bountis, and M. N. Vrahatis, How does the smaller alignment index (SALI) distinguish order from chaos? Prog. Theor. Phys. Suppl. 150, 439 (2003).

[12] Ch. Skokos, Ch. Antonopoulos, T. C. Bountis, and M. N. Vrahatis, Detecting order and chaos in Hamiltonian systems by the SALI method, J. Phys. A: Math. Gen. 37, 6269 (2004).

[13] T. C. Bountis and Ch. Skokos, Application of the SALI chaos detection method to accelerator mappings, Nucl. Instrum. Methods Phys. Res., Sect. A 561, 173 (2006).

[14] Ch. Skokos, T. C. Bountis, and Ch. Antonopoulos, Geometrical properties of local dynamics in Hamiltonian systems: The generalized alignment index (GALI) method, Physica D 231, 30 (2007).

[15] Ch. Skokos, T. C. Bountis, and Ch. Antonopoulos, Detecting chaos, determining the dimensions of tori and predicting slow diffusion in Fermi-Pasta-Ulam lattices by the generalized alignment index method, Eur. Phys. J. Spec. Top. 165, 5 (2008).

[16] P. Panagopoulos, T. C. Bountis, and Ch. Skokos, Existence and stability of localized oscillations in 1-dimensional lattices with soft-spring and hard-spring potentials, J. Vib. Acoust. 126, 520 (2004).

[17] T. Manos, Ch. Skokos, and T. Bountis, Application of the generalized alignment index (GALI) method to the dynamics of multi-dimensional symplectic maps, in Chaos, Complexity and Transport: Theory and Applications, in Proceedings of the CCT 07, edited by C. Chandre, X. Leoncini, and G. Zaslavsky (World Scientific, Singapore, 2008), pp. 356-364.

[18] Ch. Skokos and T. C. Bountis, Complex Hamiltonian Dynamics, Springer Series in Synergetics (Springer, New York, 2012).

[19] N. Voglis, M. Harsoula, and G. Contopoulos, Orbital structure in barred galaxies, Mon. Not. R. Astron. Soc. 381, 757 (2007).

[20] G. Voyatzis, Chaos, order, and periodic orbits in 3:1 resonant planetary dynamics, Astrophys. J. 675, 802 (2008).

[21] T. Bountis and K. E. Papadakis, The stability of vertical motion in the $N$-body circular Sitnikov problem, Celestial Mech. Dyn. Astron. 104, 205 (2009).

[22] T. Manos and E. Athanassoula, Regular and chaotic orbits in barred galaxies. I. Applying the SALI/GALI method to explore their distribution in several models, Mon. Not. R. Astron. Soc. 415, 629 (2011).

[23] T. Manos, T. Bountis, and Ch. Skokos, Interplay between chaotic and regular motion in a time-dependent barred galaxy model, J. Phys. A: Math. Theor. 46, 254017 (2013).

[24] N. P. Maffione, L. A. Darriba, P. M. Cincotta, and C. M. Giordano, A comparison of different indicators of chaos based on the deviation vectors: Application to symplectic mappings, Celestial Mech. Dyn. Astron. 111, 285 (2011).

[25] E. E. Zotos and N. D. Caranicolas, Order and chaos in a new 3D dynamical model describing motion in non-axially symmetric galaxies, Nonlinear Dynam. 74, 1203 (2013).

[26] E. E. Zotos, Classifying orbits in galaxy models with a prolate or an oblate dark matter halo component, Astron. Astrophys. 563, A19 (2014).

[27] N. Kyriakopoulos, V. Koukouloyannis, C. Skokos, and P. Kevrekidis, Chaotic behavior of three interacting vortices in a confined Bose-Einstein condensate, Chaos 24, 024410 (2014).
[28] Chr. Antonopoulos, V. Basios, and Tassos Bountis, Weak chaos and the "melting transition" in a confined microplasma system, Phys. Rev. E 81, 016211 (2010).

[29] P. Benitez, J. C. Losada, R. M. Benito, and F. Borondo, Analysis of the full vibrational dynamics of the LiNC/LiCN molecular system, in Progress and Challenges in Dynamical Systems, edited by S. Ibañez, J. S. Pérez del Rio, A. Pumariño, and J. A. Rodríguez, Springer Proceedings in Mathematics \& Statistics, Vol. 54 (Springer-Verlag, Berlin, 2013).

[30] A. N. Kolmogorov, Preservation of conditionally periodic movements with small change in the Hamilton function, Dokl. Akad. Nauk SSSR 98, 527 (1954) [Lect. Notes Phys. 93, 51 (1977)].

[31] P. Le Calvez and J. Wang, Some remarks on the PoincaréBirkhoff theorem, Proc. Am. Math. Soc. 138, 703 (2010).

[32] B. V. Chirikov, A universal instability of many-dimensional oscillator systems, Phys. Rep. 52, 265 (1979).

[33] M. J. Davis and S. K. Gray, Unimolecular reactions and phase space bottlenecks, J. Chem. Phys. 84, 5389 (1986).

[34] V. Afraimovich and G. M. Zaslavsky, in Kinetics and Nonlinear Dynamics in Fluids and Plasmas (Springer-Verlag, Berlin, 1998), pp. 59-82.

[35] F. Borondo, J. M. Gomez-Llorente, and R. M. Benito, Theoretical methods for the analysis of spectra of highly vibrationally excited polyatomic molecules, Laser Chem. 12, 85 (1992); F. Borondo and R. M. Benito, Dynamics and spectroscopy of highly excited molecules, in Frontiers of Chemical Dynamics, NATO ASI Series C, edited by E. Yurtsever (Kluwer, Dordrecht, 1995); F. J. Arranz, F. Borondo, and R. M. Benito, Transition from order to chaos in molecular wave functions and spectra, J. Chem. Phys. 104, 6401 (1996); Avoided crossings, scars, and transition to chaos, ibid. 107, 2395 (1997); Probability density distributions in phase space, J. Mol. Struct. Theochem 426, 87 (1998); R. M. Benito and F. Borondo, Periodic orbits and quantum mechanics of molecular Hamiltonian systems, in Ref. [5], pp. 310-313; F. Borondo, E. G. Vergini, A. A. Zembekov, and R. M. Benito, Homoclinic motions in the vibrational spectra of floppy systems: The LiCN molecule, J. Chem. Phys. 122, 111101 (2005).

[36] R. M. Benito, F. Borondo, J. H. Kim, B. G. Sumpter, and G. S. Ezra, Comparison of classical and quantum phase space structure of nonrigid molecules, LiCN, Chem. Phys. Lett. 161, 60 (1989).

[37] F. J. Arranz, F. Borondo, and R. M. Benito, Scar formation at the edge of the chaotic region, Phys. Rev. Lett. 80, 944 (1998).

[38] G. Murgida, D. Wisniacki, P. Tamborenea, and F. Borondo, Control of chemical reactions using external electric fields: The case of the LiNC $\rightleftharpoons \mathrm{LiCN}$ isomerization, Chem. Phys. Lett. 496, 356 (2010); G. Murgida, F. J. Arranz, and F. Borondo, Quantum control of isomerization by robust navigation in the energy spectrum (unpublished).

[39] P. M. Poggi, F. J. Arranz, R. M. Benito, F. Borondo, and D. A. Wisniacki, Maximum population transfer in a periodically driven quantum system, Phys. Rev. A 90, 062108 (2014).

[40] L. A. Pellouchoud and E. J. Reed, Coherent chemistry with THz pulses: Ultrafast field-driven isomerization of LiNC, Phys. Rev. A 91, 052706 (2015).

[41] P. Garcia-Muller, R. M. Benito, R. Hernandez, and F. Borondo, The role of the $\mathrm{CN}$ vibration in the activated dynamics of 
LiNC $\rightleftharpoons \mathrm{LiCN}$ isomerization in an argon solvent at high temperatures, J. Chem. Phys. 141, 074312 (2014).

[42] R. Essers, J. Tennyson, and P. E. S. Wormer, An SCF potential energy surface for lithium cyanide, Chem. Phys. Lett. 89, 223 (1982).

[43] J. C. Butcher, Numerical Methods for Ordinary Differential Equations (John Wiley \& Sons, New York, 2008).

[44] C. W. Gear, Numerical Initial Value Problems in Ordinary Differential Equations (Prentice-Hall, Englewood Cliffs, NJ, 1971).

[45] E. Hairer, S. P. Norsett, and G. Wanner, Solving Ordinary Differential Equations. I. Nonstiff Problems (Springer-Verlag, Berlin, 1993).

[46] F. Borondo, A. A. Zembekov, and R. M. Benito, Quantum manifestations of saddle-node bifurcations, Chem. Phys. Lett. 246, 421 (1995); Saddle-node bifurcations in the LiNC/LiCN molecular system: Classical aspects and quantum manifestations, J. Chem. Phys. 105, 5068 (1996); Semiclassical quantization of fragmented tori: Application to saddle-node states of LiNC/LiCN, ibid. 107, 7934 (1997).

[47] J. C. Losada, C. G. Giralda, R. M. Benito, and F. Borondo, Global dynamics of nonrigid triatomic molecular systems of three degrees of freedom, in Frontiers of Fundamental Physics: Eighth International Symposium FFP8, October 2006, Madrid, edited by B. G. Sidharth, A. Alfonso-Faus, and M. J. Fullana, AIP Conf. Proc. No. 905 (AIP, New York, 2007), p. 249.

[48] J. C. Losada, R. M. Benito, and F. Borondo, Frequency map analysis of the $3 \mathrm{D}$ vibrational dynamics of the LiCN/LiNC molecular system, Eur. Phys. J. Spec. Top. 165, 183 (2008); A. M. Tarquis, J. C. Losada, R. M. Benitoan, and F. Borondo, Multifractal analysis of tori destruction in a molecular Hamiltonian system, Phys. Rev. E 65, 016213 (2002).

[49] R. Seydel, Practical Bifurcation and Stability Analysis, Interdisciplinary Applied Mathematics 5 (Springer-Verlag, Berlin, 2010).

[50] R. Prosmiti, S. C. Farantos, R. Guantes, F. Borondo, and R. M. Benito, A periodic orbit analysis of the vibrationally highly excited LiNC/LiCN: A comparison with quantum mechanics, J. Chem. Phys. 104, 2921 (1996).

[51] F. J. Arranz, R. M. Benito, and F. Borondo, The onset of chaos in the vibrational dynamics of LiNC/LiCN, J. Chem. Phys. 123, 134305 (2005). 